\title{
Proper efficiency and duality for a new class of nonconvex multitime multiobjective variational problems
}

\author{
Ariana Pitea ${ }^{1 *}$ and Tadeusz Antczak ${ }^{2}$
}

\author{
"Correspondence: \\ arianapitea@yahoo.com \\ 'Faculty of Applied Sciences, \\ University 'Politehnica' of Bucharest, \\ Splaiul Independenţei, No. 313, \\ Bucharest, 060042, Romania \\ Full list of author information is \\ available at the end of the article
}

\begin{abstract}
In this paper, a new class of generalized of nonconvex multitime multiobjective variational problems is considered. We prove the sufficient optimality conditions for efficiency and proper efficiency in the considered multitime multiobjective variational problems with univex functionals. Further, for such vector variational problems, various duality results in the sense of Mond-Weir and in the sense of Wolfe are established under univexity. The results established in the paper extend and generalize results existing in the literature for such vector variational problems. MSC: 65K10; 90C29; 90C30

Keywords: multitime variational problem; univex function; proper efficient solution; optimality conditions; duality
\end{abstract}

\section{Introduction}

Multiobjective variational problems are very prominent amongst constrained optimization models because of their occurrences in a variety of popular contexts, notably, economic planning, advertising investment, production and inventory, epidemic, control of a rocket, etc.; for an excellent survey, see [1] Chinchuluun and Pardalos.

Several classes of functions have been defined for the purpose of weakening the limitations of convexity in mathematical programming, and also for multiobjective variational problems. Several authors have contributed in this direction: [2] Aghezzaf and Khazafi, [3] Ahmad and Sharma, [4] Arana-Jiménez et al., [5] Bector and Husain, [6] Bhatia and Mehra, [7] Hachimi and Aghezzaf, [8] Mishra and Mukherjee, [9-11] Nahak and Nanda, and others.

One class of such multiobjective optimization problems is the class of vector PDI\&PDEconstrained optimization problems in which partial differential inequalities or/and equations represent a multitude of natural phenomena of some applications in science and engineering. The areas of research which strongly motivate the PDI\&PDE-constrained optimization include: shape optimization in fluid mechanics and medicine, optimal control of processes, structural optimization, material inversion - in geophysics, data assimilation in regional weather prediction modeling, etc. PDI\&PDE-constrained optimization problems are generally infinite dimensional in nature, large and complex, [12] Chinchuluun et al.

@ 2014 Pitea and Antczak; licensee Springer. This is an Open Access article distributed under the terms of the Creative Commons Attribution License (http://creativecommons.org/licenses/by/2.0), which permits unrestricted use, distribution, and reproduction in any medium, provided the original work is properly cited. 
The basic optimization problems of path-independent curvilinear integrals with PDE constraints or with isoperimetric constraints, expressed by the multiple integrals or pathindependent curvilinear integrals, were stated for the first time by Udriște and Țevy in [13]. Later, optimality and duality results for PDI\&PDE-constrained optimization problems were established by Pitea et al. in [14] and [15].

Recently, nonconvex optimization problems with the so-called class of univex functions have been the object of increasing interest, both theoretical and applicative, and there exists nowadays a wide literature. This class of generalized convex functions was introduced in nonlinear scalar optimization problems by Bector et al. [16] as a generalization of the definition of an invex function introduced by Hanson [17]. Later, Antczak [18] used the introduced $\eta$-approximation approach for nonlinear multiobjective programming problems with univex functions to obtain new sufficient optimality conditions for such a class of nonconvex vector optimization problems. In [19], Popa and Popa defined the concept of $\rho$-univexity as a generalization univexity and $\rho$-invexity. Mishra et al. [20] established some sufficiency results for multiobjective programming problems using Lagrange multiplier conditions, and under various types of generalized $V$-univexity type-I requirements, they proved weak, strong and converse duality theorems. In [21], Khazafi and Rueda established sufficient optimality conditions and mixed type duality results under generalized $V$-univexity type I conditions for multiobjective variational programming problems.

In this paper, we study a new class of nonconvex multitime multiobjective variational problems of minimizing a vector-valued functional of curvilinear integral type. In order to prove the main results in the paper, we introduce the definition of univexity for a vectorial functional of curvilinear integral type. Thus, we establish the sufficient optimality conditions for a proper efficiency in the multitime multiobjective variational problem under univexity assumptions imposed on the functionals constituting such vector variational problems. Further, we define the multiobjective variational dual problems in the sense of Mond-Weir and in the sense of Wolfe, and we prove several dual theorems under suitable univex assumptions. The results are established for a multitime multiobjective variational problem, in which involved functions are univex with respect to the same function $\Phi$, but not necessarily with respect to the same function $b$.

\section{Preliminaries and definitions}

The following convention for equalities and inequalities will be used in the paper.

For any $x=\left(x_{1}, x_{2}, \ldots, x_{n}\right)^{T}, y=\left(y_{1}, y_{2}, \ldots, y_{n}\right)^{T}$, we define:

(i) $x=y$ if and only if $x_{i}=y_{i}$ for all $i=1,2, \ldots, n$;

(ii) $x>y$ if and only if $x_{i}>y_{i}$ for all $i=1,2, \ldots, n$;

(iii) $x \geqq y$ if and only if $x_{i} \geqq y_{i}$ for all $i=1,2, \ldots, n$;

(iv) $x \geq y$ if and only if $x \geqq y$ and $x \neq y$.

Let $(T ; h)$ and $(M ; g)$ be Riemannian manifolds of dimensions $p$ and $n$, respectively. The local coordinates on $T$ and $M$ will be written $t=\left(t^{\alpha}\right), \alpha=1, \ldots, p$ and $x=\left(x^{i}\right), i=1, \ldots, n$, respectively.

Further, let $J^{1}(T, M)$ be the first order jet bundle associated to $T$ and $M$.

Using the product order relation on $\mathbb{R}^{p}$, the hyperparallelepiped $\Omega_{t_{0}, t_{1}}$ in $\mathbb{R}^{p}$, with diagonal opposite points $t_{0}=\left(t_{0}^{1}, \ldots, t_{0}^{p}\right)$ and $t_{1}=\left(t_{1}^{1}, \ldots, t_{1}^{p}\right)$, can be written as being the interval $\left[t_{0}, t_{1}\right]$. Assume that $\gamma_{t_{0}, t_{1}}$ is a piecewise $C^{1}$-class curve joining the points $t_{0}$ and $t_{1}$. 
By $C^{\infty}\left(\Omega_{t_{0}, t_{1}}, M\right)$ we denote the space of all functions $x: \Omega_{t_{0}, t_{1}} \rightarrow M$ of $C^{\infty}$-class with the norm

$$
\|x\|=\|x\|_{\infty}+\sum_{\alpha=1}^{p}\left\|x_{\alpha}\right\|_{\infty}
$$

Now, we introduce the closed Lagrange 1-form density of $C^{\infty}$-class as follows:

$$
f_{\alpha}=\left(f_{\alpha}^{i}\right): J^{1}(T, M) \rightarrow \mathbb{R}^{r}, \quad i=1, \ldots, r, \alpha=1, \ldots, p,
$$

which determines the following path-independent curvilinear functionals:

$$
F^{i}(x(\cdot))=\int_{\gamma_{t_{0}, t_{1}}} f_{\alpha}^{i}\left(\pi_{x}(t)\right) d t^{\alpha}, \quad i=1, \ldots, r
$$

where $\pi_{x}(t)=\left(t, x(t), x_{\gamma}(t)\right)$ and $x_{\gamma}(t)=\frac{\partial x}{\partial t^{\gamma}}(t), \gamma=1, \ldots, p$, are partial velocities.

The closedness conditions (complete integrability conditions) are $D_{\beta} f_{\alpha}^{i}=D_{\alpha} f_{\beta}^{i}$ and $D_{\alpha} f_{\beta}^{i}=D_{\beta} f_{\alpha}^{i}, \alpha, \beta=1, \ldots, p, \alpha \neq \beta, i=1, \ldots, r$, where $D_{\beta}$ is the total derivative.

The following result is useful to prove the main results in the paper.

Lemma 2.1 ([22]) A total divergence is equal to a total derivative.

We also accept that the Lagrange matrix density

$$
g=\left(g_{a}^{j}\right): J^{1}\left(\Omega_{t_{0}, t_{1}}, M\right) \rightarrow \mathbb{R}^{m s}, \quad a=1, \ldots, s, j=1, \ldots, m, m<n,
$$

of $C^{\infty}$-class defines the partial differential inequalities (PDI) (of evolution)

$$
g\left(\pi_{x}(t)\right) \leqq 0, \quad t \in \Omega_{t_{0}, t_{1}},
$$

and the Lagrange matrix density

$$
h=\left(h_{a}^{l}\right): J^{1}\left(\Omega_{t_{0}, t_{1}}, M\right) \rightarrow \mathbb{R}^{k s}, \quad a=1, \ldots, s, l=1, \ldots, k, k<n,
$$

defines the partial differential equalities (PDE) (of evolution)

$$
h\left(\pi_{x}(t)\right)=0, \quad t \in \Omega_{t_{0}, t_{1}} .
$$

In the paper, consider the vector of path-independent curvilinear functionals defined by

$$
F(x(\cdot))=\int_{\gamma_{t_{0}, t_{1}}} f_{\alpha}\left(\pi_{x}(t)\right) d t^{\alpha}=\left(F^{1}(x(\cdot)), \ldots, F^{r}(x(\cdot))\right) .
$$

Denote by

$$
\begin{aligned}
\Gamma\left(\Omega_{t_{0}, t_{1}}\right)= & \left\{x(t) \in C^{\infty}\left(\Omega_{t_{0}, t_{1}}, M\right): t \in \Omega_{t_{0}, t_{1}}, x\left(t_{0}\right)=x_{0}, x\left(t_{1}\right)=x_{1},\right. \\
& \left.g\left(\pi_{x}(t)\right) \leqq 0, h\left(\pi_{x}(t)\right)=0\right\}
\end{aligned}
$$


the set all feasible solutions of problem (MVP), multitime multiobjective variational problem, introduced right now:

$$
\left\{\begin{array}{l}
\min F(x(\cdot)) \\
\text { subject to } x(\cdot) \in \Gamma\left(\Omega_{t_{0}, t_{1}}\right) .
\end{array}\right.
$$

Multiobjective programming is the search for a solution that best manages trade-offs criteria that conflict and that cannot be converted to a common measure. An optimal solution to a multiobjective programming problem is ordinarily chosen from the set of all efficient solutions (Pareto optimal solutions) to it. Therefore, for multiobjective programming problems minimization means, in general, obtaining efficient solutions (Pareto optimal solutions) in the following sense.

Definition 2.1 A feasible solution $\bar{x}(\cdot) \in \Gamma\left(\Omega_{t_{0}, t_{1}}\right)$ is called an efficient solution for problem (MVP) if there is no other feasible solution $x(\cdot) \in \Gamma\left(\Omega_{t_{0}, t_{1}}\right)$ such that

$$
F(x(\cdot)) \leq F(\bar{x}(\cdot))
$$

In other words, a feasible solution $\bar{x}(\cdot) \in \Gamma\left(\Omega_{t_{0}, t_{1}}\right)$ is called an efficient solution for problem (MVP) if there is no other feasible solution $x(\cdot) \in \Gamma\left(\Omega_{t_{0}, t_{1}}\right)$ such that

$$
\int_{\gamma_{t_{0}, t_{1}}} f_{\alpha}^{i}\left(\pi_{x}(t)\right) d t^{\alpha} \leqq \int_{\gamma_{t_{0}, t_{1}}} f_{\alpha}^{i}\left(\pi_{\bar{x}}(t)\right) d t^{\alpha}, \quad i=1, \ldots, r
$$

and

$$
\int_{\gamma_{t_{0}, t_{1}}} f_{\alpha}^{i^{*}}\left(\pi_{x}(t)\right) d t^{\alpha}<\int_{\gamma_{t_{0}, t_{1}}} f_{\alpha}^{i^{*}}\left(\pi_{\bar{x}}(t)\right) d t^{\alpha} \quad \text { for some } i^{*} \in\{1, \ldots, r\} .
$$

By normal efficient solution we understand an efficient solution to the constraint problem which is not efficient for the corresponding program without taking into consideration the constraints.

Geoffrion [23] introduced the definition of properly efficient solution in order to eliminate the efficient solutions causing unbounded trade-offs between objective functions.

Definition 2.2 A feasible solution $\bar{x}(\cdot) \in \Gamma\left(\Omega_{t_{0}, t_{1}}\right)$ is called a properly efficient solution for problem (MVP) if it is efficient for (MVP) and if there exists a positive scalar $M$ such that for all $i=1, \ldots, r$,

$$
\begin{aligned}
& \int_{\gamma_{t_{0}, t_{1}}} f_{\alpha}^{i}\left(\pi_{\bar{x}}(t)\right) d t^{\alpha}-\int_{\gamma_{t_{0}, t_{1}}} f_{\alpha}^{i}\left(\pi_{x}(t)\right) d t^{\alpha} \\
& \quad \leqq M\left(\int_{\gamma_{t_{0}, t_{1}}} f_{\alpha}^{j}\left(\pi_{x}(t)\right) d t^{\alpha}-\int_{\gamma_{t_{0}, t_{1}}} f_{\alpha}^{j}\left(\pi_{\bar{x}}(t)\right) d t^{\alpha}\right),
\end{aligned}
$$

for some $j$ such that

$$
\int_{\gamma_{t_{0}, t_{1}}} f_{\alpha}^{j}\left(\pi_{x}(t)\right) d t^{\alpha}>\int_{\gamma_{t_{0}, t_{1}}} f_{\alpha}^{j}\left(\pi_{\bar{x}}(t)\right) d t^{\alpha},
$$


whenever $x(\cdot) \in \Gamma\left(\Omega_{t_{0}, t_{1}}\right)$ and

$$
\int_{\gamma_{t_{0}, t_{1}}} f_{\alpha}^{i}\left(\pi_{x}(t)\right) d t^{\alpha}<\int_{\gamma_{t_{0}, t_{1}}} f_{\alpha}^{i}\left(\pi_{\bar{x}}(t)\right) d t^{\alpha}
$$

The following conditions established by Pitea et al. [14] are necessary for a feasible solution $\bar{x}(\cdot) \in \Gamma\left(\Omega_{t_{0}, t_{1}}\right)$ to be efficient in problem (MVP).

Theorem 2.1 Let $\bar{x}(\cdot) \in \Gamma\left(\Omega_{t_{0}, t_{1}}\right)$ be a normal efficient solution in the multitime multiobjective variational problem (MVP). Then there exist two vectors $\bar{\lambda} \in \mathbb{R}^{r}$ and the smooth matrix functions $\bar{\mu}(t)=\left(\bar{\mu}_{\alpha}(t)\right): \Omega_{t_{0}, t_{1}} \rightarrow \mathbb{R}^{\mathrm{msp}}, \bar{\xi}(t)=\left(\bar{\xi}_{\alpha}(t)\right): \Omega_{t_{0}, t_{1}} \rightarrow \mathbb{R}^{\mathrm{ksp}}$ such that

$$
\begin{aligned}
& \left\langle\bar{\lambda}, \frac{\partial f_{\alpha}}{\partial x}\left(\pi_{\bar{x}}(t)\right)\right\rangle+\left\langle\bar{\mu}_{\alpha}(t), \frac{\partial g}{\partial x}\left(\pi_{\bar{x}}(t)\right)\right\rangle+\left\langle\bar{\xi}_{\alpha}(t), \frac{\partial h}{\partial x}\left(\pi_{\bar{x}}(t)\right)\right\rangle \\
& \quad-D_{\gamma}\left(\left\langle\bar{\lambda}, \frac{\partial f_{\alpha}}{\partial x_{\gamma}}\left(\pi_{\bar{x}}(t)\right)\right\rangle+\left\langle\bar{\mu}_{\alpha}(t), \frac{\partial g}{\partial x_{\gamma}}\left(\pi_{\bar{x}}(t)\right)\right\rangle+\left\langle\bar{\xi}_{\alpha}(t), \frac{\partial h}{\partial x_{\gamma}}\left(\pi_{\bar{x}}(t)\right)\right\rangle\right) \\
& =0, \quad t \in \Omega_{t_{0}, t_{1}}, \alpha=1, \ldots, p(\text { Euler-Lagrange PDEs }), \\
& \left\langle\bar{\mu}_{\alpha}(t), g\left(\pi_{\bar{x}}(t)\right)\right\rangle=0, \quad t \in \Omega_{t_{0}, t_{1}}, \alpha=1, \ldots, p, \\
& \bar{\lambda} \geq 0, \quad\langle\bar{\lambda}, e\rangle=1, \quad \bar{\mu}_{\alpha}(t) \geqq 0, \quad t \in \Omega_{t_{0}, t_{1}}, \alpha=1, \ldots, p,
\end{aligned}
$$

where $e=(1, \ldots, 1) \in \mathbb{R}^{r}$.

We remark that relations (1) and (2) and the last relation in (3) hold true also for an efficient solution.

\section{Proper efficiency results}

Let $A: J^{1}\left(\Omega_{t_{0}, t_{1}}, M\right) \times J^{1}\left(\Omega_{t_{0}, t_{1}}, M\right) \times \mathbb{R}^{n} \rightarrow \mathbb{R}^{r}$ be a path-independent curvilinear vector functional

$$
A(x(\cdot))=\int_{\gamma_{t_{0}, t_{1}}} a_{\alpha}\left(\pi_{x}(t)\right) d t^{\alpha} .
$$

We shall introduce a definition of univexity of the above functional, which will be useful to state the results established in the paper.

Let $S$ be a nonempty subset of $C^{\infty}\left(\Omega_{t_{0}, t_{1}}, M\right), \bar{x}(\cdot) \in S$ be given, $b:=\left(b_{1}, \ldots, b_{r}\right)$ be a vector function such that $b_{i}: C^{\infty}\left(\Omega_{t_{0}, t_{1}}, M\right) \times C^{\infty}\left(\Omega_{t_{0}, t_{1}}, M\right) \rightarrow[0, \infty), i=1, \ldots, r$, and $\eta: J^{1}\left(\Omega_{t_{0}, t_{1}}, M\right) \times J^{1}\left(\Omega_{t_{0}, t_{1}}, M\right) \rightarrow \mathbb{R}^{n}$ be an $n$-dimensional vector-valued function, vanishing at the point $\left(\pi_{\bar{x}}(t), \pi_{\bar{x}}(t)\right)$, and $\Phi: \mathbb{R} \rightarrow \mathbb{R}$.

Definition 3.1 The vectorial functional $A$ is called (strictly) univex at the point $\bar{x}(\cdot)$ on $S$ with respect to $\Phi, \eta$ and $b$ if, for each $i=1, \ldots, r$, the following inequality

$$
\begin{aligned}
b_{i}(x(\cdot), \bar{x}(\cdot)) \Phi\left(A^{i}(x(\cdot))-A^{i}(\bar{x}(\cdot))\right)(>) \geqq & \int_{\gamma_{t_{0}, t_{1}}}\left\{\left\langle\eta\left(\pi_{x}(t), \pi_{\bar{x}}(t)\right), \frac{\partial a_{\alpha}^{i}}{\partial x}\left(\pi_{\bar{x}}(t)\right)\right\rangle\right. \\
& \left.+\left\langle D_{\gamma} \eta\left(\pi_{x}(\cdot), \pi_{\bar{x}}(t)\right), \frac{\partial a_{\alpha}^{i}}{\partial x_{\gamma}}\left(\pi_{\bar{x}}(t)\right)\right\rangle\right\} d t^{\alpha}
\end{aligned}
$$

holds for all $x(\cdot)$ with $x(\cdot) \neq \bar{x}(\cdot)$. 
Example 3.1 In the following, $x, \bar{x}, u:[0,1] \rightarrow \mathbb{R}$ are functions of $C^{\infty}$-class on $[0,1]$.

Let $a(x)=-x^{3}(t)$. The functional $A(x(t))=\int_{0}^{1} a\left(\pi_{x}(t)\right) d t$ is called invex at $\bar{x}(t)$ with respect to $\eta$ if

$$
A(x(t))-A(\bar{x}(t)) \geq \int_{0}^{1} \eta\left(\pi_{x}(t), \pi_{\bar{x}}(t)\right) \frac{\partial a}{\partial x}(\bar{x}(t)) d t .
$$

$A$ is univex at $\bar{x}(t)$ with respect to $\phi, \eta$ and $b$ if

$$
b(x(t), \bar{x}(t)) \phi(A(x(t))-A(\bar{x}(t))) \geq \int_{0}^{1} \eta\left(\pi_{x}(t), \pi_{\bar{x}}(t)\right) \frac{\partial a}{\partial x}(\bar{x}(t)) d t .
$$

Clearly, any invex function is univex.

We consider $b=1$.

The functional $A(x(t))=\int_{0}^{1} a(x(t)) d t$ is not invex at $\bar{x}(t)=t$ with respect to

$$
\eta\left(\pi_{x}(t), \pi_{u}(t)\right)= \begin{cases}u(t)-x(t), & \text { if } x(t)<u(t) \\ 0, & \text { otherwise. }\end{cases}
$$

Indeed, consider $x(t)=\frac{3}{2} t$. We get

$$
\begin{aligned}
& A(x(t))-A(\bar{x}(t))=\int_{0}^{1}\left(t^{3}-\frac{27}{8} t^{3}\right) d t=-\frac{19}{32} \\
& \int_{0}^{1} \eta\left(\pi_{x}(t), \pi_{\bar{x}}(t)\right) \frac{\partial a}{\partial x}(\bar{x}(t)) d t=0
\end{aligned}
$$

so the invexity condition is not satisfied.

If we take $\phi(t)=t^{2}$, we obtain that $A$ is univex with respect to $\phi, \eta$, and $b=1$, as follows:

$$
\begin{aligned}
& \phi(A(x(t))-A(\bar{x}(t)))=(A(x(t))-A(\bar{x}(t)))^{2} \geq 0 \\
& \int_{0}^{1} \eta\left(\pi_{x}(t), \pi_{\bar{x}}(t)\right) \frac{\partial a}{\partial x}(\bar{x}(t)) d t=\int_{0}^{1} \eta\left(\pi_{x}(t), \pi_{\bar{x}}(t)\right)\left(-3 \bar{x}^{2}(t)\right) d t,
\end{aligned}
$$

which is always negative since $\eta\left(\pi_{x}(t), \pi_{\bar{x}}(t)\right) \geq 0$.

Following this idea, non-invex functions for which the right-hand part of the invexity condition is negative become univex functions with the preservation of the same function $\eta$. The preservation of function $\eta$ is important when we deal with several functionals which have to be univex with respect to the same $\eta$.

Now, we prove the sufficiency of efficiency for the feasible solution $\bar{x}(\cdot) \in \Gamma\left(\Omega_{t_{0}, t_{1}}\right)$ in problem (MVP) at which the above necessary optimality conditions are fulfilled. In order to prove this result, we use the concept of univexity defined above for a vectorial functional.

Theorem 3.1 Let $\bar{x}(\cdot) \in \Gamma\left(\Omega_{t_{0}, t_{1}}\right)$ be a feasible solution in the considered multitime multiobjective variational problem (MVP), and let the necessary optimality conditions (1)-(3) be satisfied at $\bar{x}(\cdot)$. Further, assume that the following hypotheses are fulfilled: 
(a) $F^{i}(x(\cdot)), i=1, \ldots, r$, is strictly univex at the point $\bar{x}(\cdot)$ on $\Gamma\left(\Omega_{t_{0}, t_{1}}\right)$ with respect to $\Phi_{F^{i}}$, $\eta$ and $b_{F^{i}}$

(b) $\left\langle\bar{\mu}_{\alpha j}(\cdot), g^{j}(x(\cdot))\right\rangle, j=1, \ldots, m$, is univex at the point $\bar{x}(\cdot)$ on $\Gamma\left(\Omega_{t_{0}, t_{1}}\right)$ with respect to $\Phi_{g^{j}}, \eta$ and $b_{g^{j}}$,

(c) $\left\langle\bar{\xi}_{\alpha l}(\cdot), h^{l}(x(\cdot))\right\rangle, l=1, \ldots, k$, is univex at the point $\bar{x}(\cdot)$ on $\Gamma\left(\Omega_{t_{0}, t_{1}}\right)$ with respect to $\Phi_{h^{l}}$, $\eta$ and $b_{h^{l}}$,

(d) $a<0 \Longrightarrow \Phi_{F^{i}}(a)<0, i=1, \ldots, r$, and $\Phi_{F^{i}}(0)=0$,

(e) $a \leqq 0 \Longrightarrow \Phi_{g^{j}}(a) \leqq 0, j=1, \ldots, m$,

(f) $a \leqq 0 \Longrightarrow \Phi_{h^{l}}(a) \leqq 0, l=1, \ldots, k$,

(g) $b_{F^{i}}(x(\cdot), \bar{x}(\cdot))>0, i=1, \ldots, r ; b_{g^{j}}(x(\cdot), \bar{x}(\cdot)) \geqq 0, j=1, \ldots, m ; b_{h^{l}}(x(\cdot), \bar{x}(\cdot)) \geqq 0$, $l=1, \ldots, k$.

Then $\bar{x}(\cdot)$ is efficient in problem (MVP).

Proof Suppose, contrary to the result, that $\bar{x}(\cdot)$ is not efficient in problem (MVP). Then there exists $\widetilde{x}(\cdot) \in \Gamma\left(\Omega_{t_{0}, t_{1}}\right)$ such that

$$
F(\widetilde{x}(\cdot)) \leq F(\bar{x}(\cdot))
$$

Thus, for every $i=1, \ldots, r$,

$$
F^{i}(\widetilde{x}(\cdot)) \leqq F^{i}(\bar{x}(\cdot))
$$

but for at least one $i^{*}$

$$
F^{i^{*}}(\tilde{x}(\cdot))<F^{i^{*}}(\bar{x}(\cdot))
$$

Since hypotheses (a)-(e) are fulfilled, therefore, by Definition 3.1, the following inequalities

$$
\begin{aligned}
& b_{F^{i}}(x(\cdot), \bar{x}(\cdot)) \Phi_{F^{i}}\left(F^{i}(x(\cdot))-F^{i}(\bar{x}(\cdot))\right) \\
& >\int_{\gamma_{t_{0}, t_{1}}}\left\{\left\langle\eta\left(\pi_{x}(t), \pi_{\bar{x}}(t)\right), \frac{\partial f_{\alpha}^{i}}{\partial x}\left(\pi_{\bar{x}}(t)\right)\right\rangle\right. \\
& \left.\quad+\left\langle D_{\gamma} \eta\left(\pi_{x}(t), \pi_{\bar{x}}(t)\right), \frac{\partial f_{\alpha}^{i}}{\partial x_{\gamma}}\left(\pi_{\bar{x}}(t)\right)\right\rangle\right\} d t^{\alpha},
\end{aligned}
$$

and

$$
\begin{aligned}
b_{g j}(x(\cdot), \bar{x}(\cdot)) \Phi_{g j}\left(\left\langle\bar{\mu}_{\alpha j}(\cdot), g^{j}(x(\cdot))\right\rangle-\left\langle\bar{\mu}_{\alpha j}(\cdot), g^{j}(\bar{x}(\cdot))\right\rangle\right) \\
\geqq \int_{\gamma_{t_{0}, t_{1}}}\left\{\left\langle\eta\left(\pi_{x}(t), \pi_{\bar{x}}(t)\right),\left\langle\bar{\mu}_{\alpha j}(t), \frac{\partial g^{j}}{\partial x}\left(\pi_{\bar{x}}(t)\right)\right\rangle\right\rangle\right. \\
\left.\quad+\left\langle D_{\gamma} \eta\left(\pi_{x}(t), \pi_{\bar{x}}(t)\right),\left\langle\bar{\mu}_{\alpha j}(t), \frac{\partial g^{j}}{\partial x_{\gamma}}\left(\pi_{\bar{x}}(t)\right)\right\rangle\right\rangle\right\} d t^{\alpha},
\end{aligned}
$$


and

$$
\begin{aligned}
b_{h^{l}}(x(\cdot), \bar{x}(\cdot)) \Phi_{h^{l}}\left(\left\langle\bar{\xi}_{\alpha l}(\cdot), h^{l}\left(\pi_{x}(\cdot)\right)\right\rangle-\left\langle\bar{\xi}_{\alpha l}(\cdot), h^{l}\left(\pi_{\bar{x}}(t)\right)\right\rangle\right) \\
\geqq \int_{\gamma_{t_{0}, t_{1}}}\left\{\left\langle\eta\left(\pi_{x}(t), \pi_{\bar{x}}(t)\right),\left\langle\bar{\xi}_{\alpha l}(t), \frac{\partial h^{l}}{\partial x}\left(\pi_{\bar{x}}(t)\right)\right\rangle\right\rangle\right. \\
\left.\quad+\left\langle D_{\gamma} \eta\left(\pi_{x}(t), \pi_{\bar{x}}(t)\right),\left\langle\bar{\xi}_{\alpha l}(t), \frac{\partial h_{\alpha l}}{\partial x_{\gamma}}\left(\pi_{\bar{x}}(t)\right)\right\rangle\right\rangle\right\} d t^{\alpha}
\end{aligned}
$$

are satisfied for all $x(\cdot) \in \Gamma\left(\Omega_{t_{0}, t_{1}}\right)$. Hence, they are also satisfied for $x(\cdot)=\tilde{x}(\cdot)$.

Using hypotheses (d) and (f) together with (5) and (6), we get, for every $i=1, \ldots, r$,

$$
b_{F^{i}}(\widetilde{x}(\cdot), \bar{x}(\cdot)) \Phi_{F^{i}}\left(F^{i}(\widetilde{x}(\cdot))-F^{i}(\bar{x}(\cdot))\right) \leqq 0
$$

but for at least one $i^{*}$

$$
b_{F^{i}}(\widetilde{x}(\cdot), \bar{x}(\cdot)) \Phi_{F^{i}}\left(F^{i^{*}}(\widetilde{x}(\cdot))-F^{i^{*}}(\bar{x}(\cdot))\right)<0 .
$$

Combining relation (7) for $x(\cdot)=\tilde{x}(\cdot)$ together with (10) and (11), we obtain, for every $i=1, \ldots, r$,

$$
\int_{\gamma_{t_{0}, t_{1}}}\left\{\left\langle\eta\left(\pi_{\widetilde{x}}(t), \pi_{\bar{x}}(t)\right), \frac{\partial f_{\alpha}^{i}}{\partial x}\left(\pi_{\bar{x}}(t)\right)\right\rangle+\left\langle D_{\gamma} \eta\left(\pi_{\widetilde{x}}(t), \pi_{\bar{x}}(t)\right), \frac{\partial f_{\alpha}^{i}}{\partial x_{\gamma}}\left(\pi_{\bar{x}}(t)\right)\right\rangle\right\} d t^{\alpha}<0 .
$$

Multiplying each inequality above by $\bar{\lambda}_{i}, i=1, \ldots, r$, and then adding both sides of the obtained inequalities, we get

$$
\begin{aligned}
& \int_{\gamma_{t_{0}, t_{1}}}\left\{\left\langle\eta\left(\pi_{\widetilde{x}}(t), \pi_{\bar{x}}(t)\right),\left\langle\bar{\lambda}, \frac{\partial f_{\alpha}}{\partial x}\left(\pi_{\bar{x}}(t)\right)\right\rangle\right\rangle\right. \\
&\left.+\left\langle D_{\gamma} \eta\left(\pi_{\tilde{x}}(t), \pi_{\bar{x}}(t)\right),\left\langle\bar{\lambda}, \frac{\partial f_{\alpha}}{\partial x_{\gamma}}\left(\pi_{\bar{x}}(t)\right)\right\rangle\right\rangle\right\} d t^{\alpha}
\end{aligned}
$$

$<0$.

Using $\widetilde{x}(\cdot) \in \Gamma\left(\Omega_{t_{0}, t_{1}}\right)$ together with the necessary optimality conditions (2) and (3), we get, for every $j=1, \ldots, m$,

$$
\left\langle\bar{\mu}_{\alpha j}(t), g^{j}\left(\pi_{\widetilde{x}}(t)\right)\right\rangle-\left\langle\bar{\mu}_{\alpha j}(t), g^{j}\left(\pi_{\bar{x}}(t)\right)\right\rangle \leqq 0 .
$$

By assumption, we have

$$
\Phi_{g^{j}}\left(\left\langle\bar{\mu}_{\alpha j}(t), g^{j}\left(\pi_{\widetilde{x}}(t)\right)\right\rangle-\left\langle\bar{\mu}_{\alpha j}(t), g^{j}\left(\pi_{\bar{x}}(t)\right)\right\rangle\right) \leqq 0 .
$$

Since $b_{g^{j}}(x(\cdot), \bar{x}(\cdot)) \geqq 0, j=1, \ldots, m$, then

$$
b_{g^{j}}(\tilde{x}(\cdot), \bar{x}(\cdot)) \Phi_{g^{j}}\left(\left\langle\bar{\mu}_{\alpha j}(\cdot), g^{j}\left(\pi_{\widetilde{x}}(\cdot)\right)\right\rangle-\left\langle\bar{\mu}_{\alpha j}(\cdot), g^{j}\left(\pi_{\bar{x}}(\cdot)\right)\right\rangle\right) \leqq 0 .
$$


Combining (8) for $x(\cdot)=\tilde{x}(\cdot)$ and (14), we have, for every $j=1, \ldots, m$,

$$
\begin{aligned}
& \int_{\gamma_{t_{0}, t_{1}}}\left\{\eta\left\langle\left(\pi_{\tilde{x}}(t), \pi_{\bar{x}}(t)\right),\left\langle\bar{\mu}_{\alpha j}(t), \frac{\partial g^{j}}{\partial x}\left(\pi_{\bar{x}}(t)\right)\right\rangle\right\rangle\right. \\
& \left.\quad+\left\langle D_{\gamma} \eta\left(\pi_{\tilde{x}}(t), \pi_{\bar{x}}(t)\right),\left\langle\bar{\mu}_{\alpha j}(t), \frac{\partial g^{j}}{\partial x_{\gamma}}\left(\pi_{\bar{x}}(t)\right)\right\rangle\right\rangle\right\} d t^{\alpha} \\
& \leqq 0 .
\end{aligned}
$$

Adding both sides of the inequalities above, we obtain

$$
\begin{aligned}
\int_{\gamma_{t_{0}, t_{1}}} & \left\{\eta\left\langle\left(\pi_{\tilde{x}}(t), \pi_{\bar{x}}(t)\right),\left\langle\bar{\mu}_{\alpha}(t), \frac{\partial g}{\partial x}\left(\pi_{\bar{x}}(t)\right)\right\rangle\right\rangle\right. \\
+ & \left.+\left\langle D_{\gamma} \eta\left(\pi_{\tilde{x}}(t), \pi_{\bar{x}}(t)\right),\left\langle\bar{\mu}_{\alpha}(t), \frac{\partial g}{\partial x_{\gamma}}\left(\pi_{\bar{x}}(t)\right)\right\rangle\right\rangle\right\} d t^{\alpha}
\end{aligned}
$$$$
\leqq 0 \text {. }
$$

Using $\widetilde{x}(\cdot) \in \Gamma\left(\Omega_{t_{0}, t_{1}}\right)$ and $\bar{x}(\cdot) \in \Gamma\left(\Omega_{t_{0}, t_{1}}\right)$ together with hypothesis (f) and having in mind that $b_{h^{l}}(x(\cdot), \bar{x}(\cdot)) \geqq 0, l=1, \ldots, k$, we get

$$
b_{h^{l}}(\widetilde{x}(t), \bar{x}(t)) \Phi_{h^{l}}\left(\left\langle\bar{\xi}_{\alpha j}(t), h^{l}\left(\pi_{\widetilde{x}}(t)\right)\right\rangle-\left\langle\bar{\xi}_{\alpha j}(t), h^{l}\left(\pi_{\bar{x}}(t)\right)\right\rangle\right) \leqq 0 .
$$

Combining (9) with $x(\cdot)=\tilde{x}(\cdot)$ and (16), we have, for every $l=1, \ldots, k$,

$$
\begin{aligned}
& \int_{\gamma_{t_{0}, t_{1}}}\left\{\left\langle\eta\left(\pi_{\widetilde{x}}(t), \pi_{\bar{x}}(t)\right),\left\langle\bar{\xi}_{\alpha l}(t), \frac{\partial h^{l}}{\partial x}\left(\pi_{\bar{x}}(t)\right)\right\rangle\right\rangle\right. \\
& \left.+\left\langle D_{\gamma} \eta\left(\pi_{\widetilde{x}}(t), \pi_{\bar{x}}(t)\right),\left\langle\bar{\xi}_{\alpha l}(t), \frac{\partial h_{\alpha l}}{\partial x_{\gamma}}\left(\pi_{\bar{x}}(t)\right)\right\rangle\right\rangle\right\} d t^{\alpha} \\
& \leqq 0 .
\end{aligned}
$$

Adding both sides of the inequalities above, we obtain

$$
\begin{aligned}
\int_{\gamma_{t_{0}, t_{1}}} & \left\{\left\langle\eta\left(\pi_{\widetilde{x}}(t), \pi_{\bar{x}}(t)\right),\left\langle\bar{\xi}_{\alpha}(t), \frac{\partial h}{\partial x}\left(\pi_{\bar{x}}(t)\right)\right\rangle\right\rangle\right. \\
+ & \left.\left\langle D_{\gamma} \eta\left(\pi_{\widetilde{x}}(t), \pi_{\bar{x}}(t)\right),\left\langle\bar{\xi}_{\alpha}(t), \frac{\partial h_{\alpha}}{\partial x_{\gamma}}\left(\pi_{\bar{x}}(t)\right)\right\rangle\right\rangle\right\} d t^{\alpha}
\end{aligned}
$$$$
\leqq 0 \text {. }
$$

Adding both sides of inequalities (13), (15), (17), we get

$$
\begin{aligned}
\int_{\gamma_{0}, t_{1}} & \left\{\left\langle\eta\left(\pi_{\widetilde{x}}(t), \pi_{\bar{x}}(t)\right),\left\langle\bar{\lambda}, \frac{\partial f_{\alpha}}{\partial x}\left(\pi_{\bar{x}}(t)\right)\right\rangle+\left\langle\bar{\mu}_{\alpha}(t), \frac{\partial g}{\partial x}\left(\pi_{\bar{x}}(t)\right)\right\rangle\right.\right. \\
+ & \left.\left\langle\bar{\xi}_{\alpha}(t), \frac{\partial h}{\partial x}\left(\pi_{\bar{x}}(t)\right)\right\rangle\right\rangle+\left\langle D_{\gamma} \eta\left(\pi_{\widetilde{x}}(t), \pi_{\bar{x}}(t)\right),\left\langle\bar{\lambda}, \frac{\partial f_{\alpha}}{\partial x_{\gamma}}\left(\pi_{\bar{x}}(t)\right)\right\rangle\right. \\
& \left.\left.+\left\langle\bar{\mu}_{\alpha}(t), \frac{\partial g}{\partial x_{\gamma}}\left(\pi_{\bar{x}}(t)\right)\right\rangle+\left\langle\bar{\xi}_{\alpha}(t), \frac{\partial h_{\alpha}}{\partial x_{\gamma}}\left(\pi_{\bar{x}}(t)\right)\right\rangle\right\rangle\right\} d t^{\alpha}
\end{aligned}
$$

$<0$. 
We denote

$$
\begin{aligned}
W_{\alpha}\left(\pi_{\bar{x}}(t), \bar{\lambda}, \bar{\mu}_{\alpha}(t), \bar{\xi}_{\alpha}(t)\right)= & \left\langle\bar{\lambda}, \frac{\partial f_{\alpha}}{\partial x}\left(\pi_{\bar{x}}(t)\right)\right\rangle \\
& +\left\langle\bar{\mu}_{\alpha}(t), \frac{\partial g}{\partial x}\left(\pi_{\bar{x}}(t)\right)\right\rangle+\left\langle\bar{\xi}_{\alpha}(t), \frac{\partial h}{\partial x_{\gamma}}\left(\pi_{\bar{x}}(t)\right)\right\rangle .
\end{aligned}
$$

Hence, (18) yields

$$
\begin{aligned}
& \int_{\gamma_{t_{0}, t_{1}}}\left\{\left\langle\eta\left(\pi_{\widetilde{x}}(t), \pi_{\bar{x}}(t)\right), \frac{\partial W}{\partial x}\left(\pi_{\bar{x}}(t), \bar{\lambda}, \bar{\mu}_{\alpha}(t), \bar{\xi}_{\alpha}(t)\right)\right\rangle\right. \\
& \left.\quad+\left\langle D_{\gamma} \eta\left(\pi_{\widetilde{x}}(t), \pi_{\bar{x}}(t)\right), \frac{\partial W}{\partial x_{\gamma}}\left(\pi_{\bar{x}}(t), \bar{\lambda}, \bar{\mu}_{\alpha}(t), \bar{\xi}_{\alpha}(t)\right)\right\rangle\right\} d t^{\alpha} \\
& \quad<0 .
\end{aligned}
$$

Using the following relation

$$
\begin{aligned}
\left\langle D_{\gamma} \eta\left(\pi_{\tilde{x}}(t), \pi_{\bar{x}}(t)\right), \frac{\partial W}{\partial x_{\gamma}}\left(\pi_{\bar{x}}(t), \bar{\lambda}, \bar{\mu}_{\alpha}(t), \bar{\xi}_{\alpha}(t)\right)\right\rangle \\
=D_{\gamma}\left\langle\eta\left(\pi_{\tilde{x}}(t), \pi_{\bar{x}}(t)\right), \frac{\partial W}{\partial x_{\gamma}}\left(\pi_{\bar{x}}(t), \bar{\lambda}_{,}, \bar{\mu}_{\alpha}(t), \bar{\xi}_{\alpha}(t)\right)\right\rangle \\
\quad-\left\langle\eta\left(\pi_{\widetilde{x}}(t), \pi_{\bar{x}}(t)\right), D_{\gamma}\left(\frac{\partial W}{\partial x_{\gamma}}\right)\left(\pi_{\bar{x}}(t), \bar{\lambda}, \bar{\mu}_{\alpha}(t), \bar{\xi}_{\alpha}(t)\right)\right\rangle
\end{aligned}
$$

in inequality (20), we get

$$
\begin{aligned}
\int_{\gamma_{0}, t_{1}} & \left\{\left\langle\eta\left(\pi_{x}(t), \pi_{\bar{x}}(t)\right), \frac{\partial W}{\partial x_{\alpha}}\left(\pi_{\bar{x}}(t), \bar{\lambda}, \bar{\mu}_{\alpha}(t), \bar{\xi}_{\alpha}(t)\right)\right\rangle\right. \\
& +D_{\gamma}\left\langle\eta\left(\pi_{x}(t), \pi_{\bar{x}}(t)\right), \frac{\partial W}{\partial x_{\gamma}}\left(\pi_{\bar{x}}(t), \bar{\lambda}, \bar{\mu}_{\alpha}(t), \bar{\xi}_{\alpha}(t)\right)\right\rangle \\
& \left.-\left\langle\eta\left(\pi_{x}(t), \pi_{\bar{x}}(t)\right), D_{\gamma}\left(\frac{\partial W}{\partial x_{\gamma}}\right)\left(\pi_{\bar{x}}(t), \bar{\lambda}, \bar{\mu}_{\alpha}(t), \bar{\xi}_{\alpha}(t)\right)\right\rangle\right\} d t^{\alpha}
\end{aligned}
$$

$<0$.

By Euler-Lagrange PDE (1), it follows that

$$
\int_{\gamma_{t_{0}, t_{1}}} D_{\gamma}\left\langle\eta\left(\pi_{\tilde{x}}(t), \pi_{\bar{x}}(t)\right), \frac{\partial W}{\partial x_{\gamma}}\left(\pi_{\bar{x}}(t), \bar{\lambda}, \bar{\mu}_{\alpha}(t), \bar{\xi}_{\alpha}(t)\right)\right\rangle d t^{\alpha}<0 .
$$

For $\alpha=1, \ldots, p, \gamma=1, \ldots, p$, we denote

$$
Q_{\alpha}^{\gamma}(t)=\left\langle\eta\left(\pi_{x}(t), \pi_{\bar{x}}(t)\right), \frac{\partial W}{\partial x_{\gamma}}\left(\pi_{\bar{x}}(t), \bar{\lambda}, \bar{\mu}_{\alpha}(t), \bar{\xi}_{\alpha}(t)\right)\right\rangle,
$$

and

$$
I=\int_{\gamma t_{0}, t_{1}} D_{\gamma} Q_{\alpha}^{\gamma}(t) d t^{\alpha}
$$


Combining (22), (23) and (24), we get

$$
I=\int_{\gamma_{t_{0}, t_{1}}} D_{\gamma} Q_{\alpha}^{\gamma}(t) d t^{\alpha}<0
$$

According to Lemma 2.1, it follows that there exists $Q(t)$ with $Q\left(t_{0}\right)=0$ and $Q\left(t_{1}\right)=0$ such that

$$
D_{\gamma} Q_{\alpha}^{\gamma}(t)=D_{\alpha} Q(t)
$$

Therefore, by (24) and (26), we have

$$
I=\int_{\gamma_{t_{0}, t_{1}}} D_{\alpha} Q(t) d t^{\alpha}=Q\left(t_{1}\right)-Q\left(t_{0}\right)=0,
$$

contradicting (25). This means that $\bar{x}(\cdot)$ is efficient in problem (MVP), and this completes the proof of the theorem.

Theorem 3.2 Let $\bar{x}(\cdot) \in \Gamma\left(\Omega_{t_{0}, t_{1}}\right)$ be a feasible solution in the considered multitime multiobjective variational problem (MVP), and let the necessary optimality conditions (1)-(3) be satisfied at $\bar{x}(\cdot)$. Further, assume that hypotheses (a)-(g) in Theorem 3.1 are fulfilled.

If $\bar{\lambda}>0$, then $\bar{x}(\cdot)$ is properly efficient in problem (MVP).

Proof The proof follows in a manner similar to that of Theorem 3.1.

\section{Mond-Weir type duality}

In this section, consider the vector of path-independent curvilinear functionals defined by

$$
F(y(\cdot))=\int_{\gamma_{t_{0}, t_{1}}} f_{\alpha}\left(\pi_{y}(t)\right) d t^{\alpha}=\left(F^{1}(y(\cdot)), \ldots, F^{r}(y(\cdot))\right)
$$

and define the following multiobjective dual problem in the sense of Mond-Weir for the considered multitime multiobjective variational problem (MVP):

$$
\begin{aligned}
& \min F(y(\cdot)), \quad \text { subject to } \\
& \left\langle\lambda, \frac{\partial f_{\alpha}}{\partial x}\left(\pi_{y}(t)\right)\right\rangle+\left\langle\mu_{\alpha}(t), \frac{\partial g}{\partial x}\left(\pi_{y}(t)\right)\right\rangle+\left\langle\xi_{\alpha}(t), \frac{\partial h}{\partial x}\left(\pi_{y}(t)\right)\right\rangle \\
& \quad-D_{\gamma}\left(\left\langle\lambda, \frac{\partial f_{\alpha}}{\partial x_{\gamma}}\left(\pi_{y}(t)\right)\right\rangle+\left\langle\mu_{\alpha}(t), \frac{\partial g}{\partial x_{\gamma}}\left(\pi_{y}(t)\right)\right\rangle+\left\langle\xi_{\alpha}(t), \frac{\partial h}{\partial x_{\gamma}}\left(\pi_{y}(t)\right)\right\rangle\right) \\
& \quad=0, \\
& \left\langle\mu_{\alpha}(t), g\left(\pi_{y}(t)\right\rangle+\left\langle\xi_{\alpha}(t), h\left(\pi_{y}(t)\right)\right\rangle \geqq 0, \quad t \in \Omega_{t_{0}, t_{1}}, \alpha=1, \ldots, p,\right. \\
& y\left(t_{0}\right)=y_{0}, \quad y\left(t_{1}\right)=y_{1}, \quad, \quad \mu_{\alpha}(t) \geqq 0, \quad t \in \Omega_{t_{0}, t_{1}}, \alpha=1, \ldots, p, \\
& \lambda \geq 0, \quad\langle\lambda, e\rangle=1, \quad
\end{aligned}
$$

where $e=(1, \ldots, 1) \in \mathbb{R}^{r}$ and $y_{\gamma}(t)=\frac{\partial y}{\partial t^{\gamma}}(t), \gamma=1, \ldots, p$, are partial velocities. 
Let $\Delta_{M W}\left(\Omega_{t_{0}, t_{1}}\right)$ be the set of all feasible solutions $\left(y(\cdot), y_{\gamma}(\cdot), \lambda, \mu(\cdot), \xi(\cdot)\right)$ in the MondWeir type dual problem (MWDP), that is,

$$
\begin{aligned}
\Delta_{M W}\left(\Omega_{t_{0}, t_{1}}\right)= & \left\{(y(t), \lambda, \mu(t), \xi(t)): t \in \Omega_{t_{0}, t_{1}}, y(t) \in C^{\infty}\left(\Omega_{t_{0}, t_{1}}, M\right), \lambda \in \mathbb{R}^{r},\right. \\
& \mu(t): \Omega_{t_{0}, t_{1}} \rightarrow \mathbb{R}^{\mathrm{msp}}, \xi(t): \Omega_{t_{0}, t_{1}} \rightarrow \mathbb{R}^{\mathrm{msp}} \\
& \text { verifying the constraints of (MWDP) }\} .
\end{aligned}
$$

Let $Y=\left\{y(t) \in C^{\infty}\left(\Omega_{t_{0}, t_{1}}, M\right):(y(t), \lambda, \mu(t), \xi(t)) \in \Delta_{M W}\left(\Omega_{t_{0}, t_{1}}\right)\right\}$.

Theorem 4.1 (Weak duality) Consider $x(\cdot)$ to be a feasible solution of problem (MVP) and $(y(\cdot), \lambda, \mu(\cdot), \xi(\cdot))$ to be a feasible solution of problem (MWDP).

Suppose that the following conditions are satisfied:

(a) $F^{i}(y(\cdot)), i=1, \ldots, r$, is univex at $y(\cdot)$ on $\Gamma\left(\Omega_{t_{0}, t_{1}}\right) \cup Y$ with respect to $\Phi_{F^{i}}, \eta$, and $b_{F^{i}}$;

(b) $\left\langle\mu_{\alpha}(\cdot), g\left(\pi_{y}(\cdot)\right)\right\rangle+\left\langle\xi_{\alpha}(\cdot), h\left(\pi_{y}(\cdot)\right)\right\rangle$ is univex at $y(\cdot)$ on $\Gamma\left(\Omega_{t_{0}, t_{1}}\right) \cup Y$ with respect to $\Phi$, $\eta$, and $b$;

(c) $a<0 \Longrightarrow \Phi_{F^{i}}(a)<0, i=1, \ldots, r$, and $\Phi_{F^{i}}(0)=0$;

(d) $a \leqq 0 \Longrightarrow \Phi(a) \leqq 0$;

(e) $b_{F^{i}}(x(\cdot), y(\cdot))>0, i=1, \ldots, r$.

Then the inequality $F(x(\cdot))<F(y(\cdot))$ is false.

Proof Suppose $F^{i}(x(\cdot)) \leq F^{i}(y(\cdot))$ for all $i=1, \ldots, r$. We obtain

$$
\Phi_{F^{i}}\left(F^{i}(x(\cdot))-F^{i}(y(\cdot))\right)<0, \quad i=1, \ldots, r,
$$

and using hypothesis (a) and Definition 3.1, we get

$$
\begin{aligned}
b_{F^{i}}(x(t), y(t)) \Phi_{F^{i}}\left(F^{i}(x(\cdot))-F^{i}(y(\cdot))\right)> & \int_{\gamma_{t_{0}, t_{1}}}\left\{\left\langle\eta\left(\pi_{x}(t), \pi_{y}(t)\right), \frac{\partial f_{\alpha}^{i}}{\partial x}\left(\pi_{y}(t)\right)\right\rangle\right. \\
& \left.+\left\langle D_{\gamma} \eta\left(\pi_{x}(t), \pi_{y}(t)\right), \frac{\partial f_{\alpha}^{i}}{\partial x_{\gamma}}\left(\pi_{y}(t)\right)\right\rangle\right\} d t^{\alpha} .
\end{aligned}
$$

We multiply (29) by $\lambda_{i}$ and make the sum from $i=1$ to $i=r$, obtaining

$$
\begin{aligned}
& \sum_{i=1}^{r} \lambda_{i} b_{F^{i}}(x(t), y(t)) \Phi_{F^{i}}\left(F^{i}(x(\cdot))-F^{i}(y(\cdot))\right) \\
& \leqq \\
& \quad \int_{\gamma_{t_{0}, t_{1}}}\left\{\left\langle\eta\left(\pi_{x}(t), \pi_{y}(t)\right),\left\langle\lambda, \frac{\partial f_{\alpha}}{\partial x}\left(\pi_{y}(t)\right)\right\rangle\right\rangle\right. \\
& \left.\quad+\left\langle D_{\gamma} \eta\left(\pi_{x}(t), \pi_{y}(t)\right),\left\langle\lambda, \frac{\partial f_{\alpha}}{\partial x_{\gamma}}\left(\pi_{y}(t)\right)\right\rangle\right\rangle\right\} d t^{\alpha} .
\end{aligned}
$$

According to hypothesis (c), (28) and (30) imply

$$
\begin{aligned}
0> & \int_{\gamma_{t_{0}, t_{1}}}\left\{\left\langle\eta\left(\pi_{x}(t), \pi_{y}(t)\right),\left\langle\lambda, \frac{\partial f_{\alpha}}{\partial x}\left(\pi_{y}(t)\right)\right\rangle\right\rangle\right. \\
& \left.+\left\langle D_{\gamma} \eta\left(\pi_{x}(t), \pi_{y}(t)\right),\left\langle\lambda, \frac{\partial f_{\alpha}}{\partial x_{\gamma}}\left(\pi_{y}(t)\right)\right\rangle\right\rangle\right\} d t^{\alpha} .
\end{aligned}
$$


From the feasibility of $x(\cdot)$ in the considered multitime multiobjective variational problem (MVP), it follows that

$$
\left\langle\mu_{\alpha}(t), g\left(\pi_{x}(t)\right)\right\rangle+\left\langle\xi_{\alpha}(t), h\left(\pi_{x}(t)\right)\right\rangle \leqq 0,
$$

while the feasibility of $\left(y(\cdot), y_{\gamma}(\cdot), \lambda, \mu(\cdot), \xi(\cdot)\right)$ in the considered multitime multiobjective variational problem (MWDP) gives

$$
\left\langle\mu_{\alpha}(t), g\left(\pi_{y}(t)\right)\right\rangle+\left\langle\xi_{\alpha}(t), h\left(\pi_{y}(t)\right)\right\rangle \geqq 0 .
$$

Combining (32) and (33), we obtain

$$
\left\langle\mu_{\alpha}(t), g\left(\pi_{x}(t)\right)\right\rangle+\left\langle\xi_{\alpha}(t), h\left(\pi_{x}(t)\right)\right\rangle-\left[\left\langle\mu_{\alpha}(t), g\left(\pi_{y}(t)\right)\right\rangle+\left\langle\xi_{\alpha}(t), h\left(\pi_{y}(t)\right)\right\rangle\right] \leqq 0 .
$$

According to hypothesis (d), (34) implies

$$
\Phi\left(\left\langle\mu_{\alpha}(t), g\left(\pi_{x}(t)\right)\right\rangle+\left\langle\xi_{\alpha}(t), h\left(\pi_{x}(t)\right)\right\rangle-\left[\left\langle\mu_{\alpha}(t), g\left(\pi_{y}(t)\right)\right\rangle+\left\langle\xi_{\alpha}(t), h\left(\pi_{y}(t)\right)\right\rangle\right]\right) \leqq 0 .
$$

But $b(x(t), y(t)) \geqq 0$, by consequence, the inequality above gives

$$
\begin{aligned}
& b(x(t), y(t)) \Phi\left(\left\langle\mu_{\alpha}(t), g\left(\pi_{x}(t)\right)\right\rangle+\left\langle\xi_{\alpha}(t), h\left(\pi_{x}(t)\right)\right\rangle\right. \\
& \left.\quad-\left[\left\langle\mu_{\alpha}(t), g\left(\pi_{y}(t)\right)\right\rangle+\left\langle\xi_{\alpha}(t), h\left(\pi_{y}(t)\right)\right\rangle\right]\right) \\
& \leqq 0 .
\end{aligned}
$$

Using hypothesis (b) together with Definition 3.1 and (35), we get that the inequality

$$
\begin{aligned}
\int_{\gamma_{t_{0}, t_{1}}} & \left\{\left\langle\eta\left(\pi_{x}(t), \pi_{y}(t)\right),\left\langle\mu_{\alpha}(t), \frac{\partial g}{\partial y}\left(\pi_{y}(t)\right)\right\rangle+\left\langle\xi_{\alpha}(t), \frac{\partial h}{\partial y}\left(\pi_{y}(t)\right)\right\rangle\right\rangle\right. \\
& \left.+\left\langle D_{\gamma} \eta\left(\pi_{x}(t), \pi_{y}(t)\right),\left\langle\mu_{\alpha}(t), \frac{\partial g}{\partial y_{\gamma}}\left(\pi_{y}(t)\right)\right\rangle+\left\langle\xi_{\alpha}(t), \frac{\partial h_{\alpha}}{\partial y_{\gamma}}\left(\pi_{y}(t)\right)\right\rangle\right\rangle\right\} d t^{\alpha} \\
\leqq &
\end{aligned}
$$

holds. For each $\alpha=1, \ldots, p$, we introduce

$$
V_{\alpha}\left(\pi_{y}(t), \lambda, \mu(t), \xi(t)\right)=\left\langle\lambda, f_{\alpha}\left(\pi_{y}(t)\right)\right\rangle+\left\langle\mu_{\alpha}(t), g\left(\pi_{y}(t)\right)\right\rangle+\left\langle\xi_{\alpha}(t), h\left(\pi_{y}(t)\right)\right\rangle .
$$

Adding both sides of (31) and (36) and taking into account (37), we obtain

$$
\begin{aligned}
\int_{\gamma_{0}, t_{1}} & \left\{\left\langle\eta\left(\pi_{x}(t), \pi_{y}(t)\right), \frac{\partial V_{\alpha}}{\partial y}\left(\pi_{y}(t), \lambda, \mu(t), \xi(t)\right)\right\rangle\right. \\
+ & \left.\left\langle D_{\gamma} \eta\left(\pi_{x}(t), \pi_{y}(t)\right), \frac{\partial V_{\alpha}}{\partial y_{\gamma}}\left(\pi_{y}(t), \lambda, \mu(t), \xi(t)\right)\right\rangle\right\} d t^{\alpha}
\end{aligned}
$$

$<0$. 
Using the relation

$$
\begin{aligned}
\left\langle D_{\gamma} \eta\left(\pi_{x}(t), \pi_{y}(t)\right), \frac{\partial V_{\alpha}}{\partial y_{\gamma}}\left(\pi_{y}(t), \lambda, \mu(t), \xi(t)\right)\right\rangle \\
=D_{\gamma}\left\langle\eta\left(\pi_{x}(t), \pi_{y}(t)\right), \frac{\partial V_{\alpha}}{\partial y_{\gamma}}\left(\pi_{y}(t), \lambda, \mu(t), \xi(t)\right)\right\rangle \\
\quad-\left\langle\eta\left(\pi_{x}(t), \pi_{y}(t)\right), D_{\gamma}\left(\frac{\partial V_{\alpha}}{\partial y_{\gamma}}\right)\left(\pi_{y}(t), \lambda, \mu(t), \xi(t)\right)\right\rangle
\end{aligned}
$$

together with the constraints of (MWDP), we obtain from (38) that the inequality

$$
\int_{\gamma_{t_{0}, t_{1}}} D_{\gamma}\left\langle\eta\left(\pi_{x}(t), \pi_{y}(t)\right), \frac{\partial V_{\alpha}}{\partial y_{\gamma}}\left(\pi_{y}(t), \lambda, \mu(t), \xi(t)\right)\right\rangle d t^{\alpha}<0
$$

holds. According to Lemma 2.1, we obtain that the above integral is equal to 0 , contradicting (40). This means that the inequality $F(x(\cdot))<F(y(\cdot))$ is false and completes the proof of the theorem.

If we impose some stronger assumption on the objective function, then we can prove a stronger result.

Theorem 4.2 (Strong duality) Let $\bar{x}(\cdot)$ be a normal efficient solution of (MVP). Then there exist a vector $\bar{\lambda}$ in $\mathbb{R}^{r}$ and smooth matrix functions $\bar{\mu}(t)=\left(\bar{\mu}_{\alpha}(t)\right): \Omega_{t_{0}, t_{1}} \rightarrow \mathbb{R}^{\mathrm{msp}}$ and $\bar{\xi}(t)=\left(\bar{\xi}_{\alpha}(t)\right): \Omega_{t_{0}, t_{1}} \rightarrow \mathbb{R}^{\mathrm{ksp}}$ such that $(\bar{x}(\cdot), \bar{\lambda}, \bar{\mu}(\cdot), \bar{\xi}(\cdot))$ is feasible in the Mond-Weir multitime multiobjective variational problem (MWDP) and the objective functions of (MVP) and (MWDP) are equal at these points. If also all the hypotheses of Theorem 4.1 are satisfied, and $\bar{\lambda}>0$, then $(\bar{x}(\cdot), \bar{\lambda}, \bar{\mu}(\cdot), \bar{\xi}(\cdot))$ is a properly efficient solution in (MWDP).

Proof Let $\bar{x}(\cdot)$ be a normal efficient solution in the considered multitime multiobjective variational problem (MVP). Then, by Theorem 2.1, there exist the vector $\bar{\lambda} \in \mathbb{R}^{r}$ and the smooth matrix functions $\bar{\mu}(t)=\left(\bar{\mu}_{\alpha}(t)\right): \Omega_{t_{0}, t_{1}} \rightarrow \mathbb{R}^{\mathrm{msp}}, \bar{\xi}(t)=\left(\bar{\xi}_{\alpha}(t)\right): \Omega_{t_{0}, t_{1}} \rightarrow \mathbb{R}^{\mathrm{ksp}}$ such that conditions (1)-(3) are fulfilled. Therefore, $(\bar{x}(\cdot), \bar{\lambda}, \bar{\mu}(\cdot), \bar{\xi}(\cdot))$ is feasible in (MWDP). Thus, by weak duality, it follows that $(\bar{x}(\cdot), \bar{\lambda}, \bar{\mu}(\cdot), \bar{\xi}(\cdot))$ is an efficient solution in (MWDP).

We shall prove that $(\bar{x}(\cdot), \bar{\lambda}, \bar{\mu}(\cdot), \bar{\xi}(\cdot))$ is a properly efficient solution in (MWDP) by the method of contradiction. Suppose that $(\bar{x}(\cdot), \bar{\lambda}, \bar{\mu}(\cdot), \bar{\xi}(\cdot))$ is not so. Then there exists $\widetilde{\gamma}(\cdot), \tilde{\lambda}, \tilde{\mu}(\cdot), \widetilde{\xi}(\cdot))$ feasible in (MWDP) satisfying

$$
\int_{\gamma_{t_{0}, t_{1}}} f_{\alpha}^{i}\left(\pi_{\bar{x}}(t)\right) d t^{\alpha}>\int_{\gamma_{t_{0}, t_{1}}} f_{\alpha}^{i}\left(\pi_{\tilde{y}}(t)\right) d t^{\alpha}
$$

for some $i$ such that the following inequality

$$
\begin{aligned}
& \int_{\gamma t_{0}, t_{1}} f_{\alpha}^{i}\left(\pi_{\bar{x}}(t)\right) d t^{\alpha}-\int_{\gamma t_{0}, t_{1}} f_{\alpha}^{i}\left(\pi_{\tilde{y}}(t)\right) d t^{\alpha} \\
& >M\left(\int_{\gamma_{t_{0}, t_{1}}} f_{\alpha}^{j}\left(\pi_{\tilde{y}}(t)\right) d t^{\alpha}-\int_{\gamma_{t_{0}, t_{1}}} f_{\alpha}^{j}\left(\pi_{\bar{x}}(t)\right) d t^{\alpha}\right)
\end{aligned}
$$


holds for every scalar $M>0$ and for each $j \neq i$ satisfying

$$
\int_{\gamma_{t_{0}, t_{1}}} f_{\alpha}^{j}\left(\pi_{\widetilde{y}}(t)\right) d t^{\alpha}>\int_{\gamma_{t_{0}, t_{1}}} f_{\alpha}^{j}\left(\pi_{\bar{x}}(t)\right) d t^{\alpha} .
$$

Assume that $r \geqq 2$, and then we set

$$
M=(r-1) \max _{i, j} \frac{\bar{\lambda}_{j}}{\bar{\lambda}_{i}}, \quad i \neq j, i, j=1, \ldots, r .
$$

Combining (41) and (43), we get that, for each $j \neq i$,

$$
\begin{aligned}
& \int_{\gamma_{t_{0}, t_{1}}} f_{\alpha}^{i}\left(\pi_{\bar{x}}(t)\right) d t^{\alpha}-\int_{\gamma_{t_{0}, t_{1}}} f_{\alpha}^{i}(\pi \widetilde{y}(t)) d t^{\alpha} \\
& \quad>(r-1) \max _{i, j} \frac{\bar{\lambda}_{j}}{\bar{\lambda}_{i}}\left(\int_{\gamma_{t_{0}, t_{1}}} f_{\alpha}^{j}\left(\pi_{\tilde{y}}(t)\right) d t^{\alpha}-\int_{\gamma_{t_{0}, t_{1}}} f_{\alpha}^{j}\left(\pi_{\bar{x}}(t)\right) d t^{\alpha}\right) .
\end{aligned}
$$

Thus, (42) gives

$$
\begin{aligned}
& \frac{\bar{\lambda}_{i}}{r-1} \int_{\gamma_{t_{0}, t_{1}}} f_{\alpha}^{i}\left(\pi_{\bar{x}}(t)\right) d t^{\alpha}-\int_{\gamma_{t_{0}, t_{1}}} f_{\alpha}^{i}\left(\pi_{\widetilde{y}}(t)\right) d t^{\alpha} \\
& \quad>\bar{\lambda}_{j}\left(\int_{\gamma_{t_{0}, t_{1}}} f_{\alpha}^{j}\left(\pi_{\tilde{y}}(t)\right) d t^{\alpha}-\int_{\gamma_{t_{0}, t_{1}}} f_{\alpha}^{j}\left(\pi_{\bar{x}}(t)\right) d t^{\alpha}\right) .
\end{aligned}
$$

Adding both sides of the inequalities above with respect to $j$ and taking into account that $j \neq i$, we obtain

$$
\begin{aligned}
& \bar{\lambda}_{i}\left(\int_{\gamma_{t_{0}, t_{1}}} f_{\alpha}^{i}\left(\pi_{\bar{x}}(t)\right) d t^{\alpha}-\int_{\gamma_{t_{0}, t_{1}}} f_{\alpha}^{i}\left(\pi_{\widetilde{y}}(t)\right) d t^{\alpha}\right) \\
& \quad>\sum_{j \neq i} \bar{\lambda}_{j}\left(\int_{\gamma_{t_{0}, t_{1}}} f_{\alpha}^{j}\left(\pi_{\widetilde{y}}(t)\right) d t^{\alpha}-\int_{\gamma_{t_{0}, t_{1}}} f_{\alpha}^{j}\left(\pi_{\bar{x}}(t)\right) d t^{\alpha}\right) .
\end{aligned}
$$

Thus, (42) implies that the following inequality

$$
\begin{aligned}
& \bar{\lambda}_{i} \int_{\gamma_{t_{0}, t_{1}}} f_{\alpha}^{i}\left(\pi_{\bar{x}}(t)\right) d t^{\alpha}+\sum_{j \neq i} \int_{\gamma_{t_{0}, t_{1}}} f_{\alpha}^{j}(\pi \widetilde{y}(t)) d t^{\alpha} \\
& \quad>\bar{\lambda}_{i} \int_{\gamma_{t_{0}, t_{1}}} f_{\alpha}^{i}(\pi \widetilde{y}(t)) d t^{\alpha}+\sum_{j \neq i} \bar{\lambda}_{j} \int_{\gamma_{t_{0}, t_{1}}} f_{\alpha}^{j}(\pi \widetilde{y}(t)) d t^{\alpha}
\end{aligned}
$$

holds, which is a contradiction to the efficiency of $\bar{x}(\cdot)$ in problem (MVP). This means that $(\bar{x}(\cdot), \bar{\lambda}, \bar{\mu}(\cdot), \bar{\xi}(\cdot))$ is a properly efficient solution in problem (MWDP). Hence, the proof of the theorem is complete.

Proposition 4.1 Let $(\bar{y}(\cdot), \bar{\lambda}, \bar{\mu}(\cdot), \bar{\xi}(\cdot))$ be a feasible solution in problem (MWDP) with $\bar{\lambda}>0$ and $\bar{y}(\cdot) \in \Gamma\left(\Omega_{t_{0}, t_{1}}\right)$. Assume that hypotheses (a)-(d) of Theorem 4.1 are satisfied and that condition (e) holds true for each $x(\cdot) \in \Gamma\left(\Omega_{t_{0}, t_{1}}\right)$.

Then $\bar{y}(\cdot)$ is a properly efficient solution in the considered multitime multiobjective variational problem (MVP). 
Proof The efficiency of $\bar{y}(\cdot)$ in problem (MVP) follows from the weak duality theorem. The proof of proper efficiency of $\bar{y}(\cdot)$ in (MVP) is similar to that of Theorem 4.2.

Theorem 4.3 Let $(\bar{\gamma}(\cdot), \bar{\lambda}, \bar{\mu}(\cdot), \bar{\xi}(\cdot))$ be a properly efficient solution in problem (MWDP) and $\bar{y}(\cdot) \in \Gamma\left(\Omega_{t_{0}, t_{1}}\right)$. Assume that hypotheses (a)-(d) of Theorem 4.1 are satisfied and that condition (e) holds true for each $x(\cdot) \in \Gamma\left(\Omega_{t_{0}, t_{1}}\right)$.

Then $\bar{y}(\cdot)$ is a properly efficient solution in the considered multitime multiobjective variational problem (MVP).

Proof Proof follows directly from Proposition 4.1.

\section{Wolfe type duality}

In this section, consider the functional

$$
\varphi(y(\cdot), \mu(\cdot), \xi(\cdot))=\int_{\gamma_{t_{0}, t_{1}}}\left\{f_{\alpha}\left(\pi_{y}(t)\right)+\left[\left\langle\mu_{\alpha}(t), g\left(\pi_{y}(t)\right)\right\rangle+\left\langle\xi_{\alpha}(t), h\left(\pi_{y}(t)\right)\right\rangle\right] e\right\} d t^{\alpha}
$$

and the associated multitime multiobjective variational dual problem of (MVP) in the sense of Wolfe, designated by (WDP):

$\min \varphi(y(\cdot), \mu(\cdot), \xi(\cdot))$

subject to

$$
\begin{aligned}
& \left\langle\lambda, \frac{\partial f_{\alpha}}{\partial y}\left(\pi_{y}(t)\right)\right\rangle+\left\langle\mu_{\alpha}(t), \frac{\partial g}{\partial y}\left(\pi_{y}(t)\right)\right\rangle+\left\langle\xi_{\alpha}(t), \frac{\partial h}{\partial y}\left(\pi_{y}(t)\right)\right\rangle \\
& \quad-D_{\gamma}\left(\left\langle\lambda, \frac{\partial f_{\alpha}}{\partial y_{\gamma}}\left(\pi_{y}(t)\right)\right\rangle+\left\langle\mu_{\alpha}(t), \frac{\partial g}{\partial y_{\gamma}}\left(\pi_{y}(t)\right)\right\rangle+\left\langle\xi_{\alpha}(t), \frac{\partial h}{\partial y_{\gamma}}\left(\pi_{y}(t)\right)\right\rangle\right) \\
& =0, \quad t \in \Omega_{t_{0}, t_{1}}, \\
& y\left(t_{0}\right)=y_{0}, \quad y\left(t_{1}\right)=y_{1}, \quad \\
& \lambda \geq 0, \quad\langle\lambda, e\rangle=1, \quad \mu_{\alpha}(t) \geqq 0, \quad t \in \Omega_{t_{0}, t_{1}}, \alpha=1, \ldots, p,
\end{aligned}
$$

where $e=(1, \ldots, 1)^{T} \in \mathbb{R}^{r}$ and $y_{\gamma}(t)=\frac{\partial y}{\partial t^{\gamma}}(t), \gamma=1, \ldots, p$, are partial velocities.

Let $\Delta_{W}\left(\Omega_{t_{0}, t_{1}}\right)$ be the set of all feasible solutions $\left(y(\cdot), y_{\gamma}(\cdot), \lambda, \mu(\cdot), \xi(\cdot)\right)$ in the Wolfe type dual problem (WDP), that is,

$$
\begin{aligned}
\Delta_{W}\left(\Omega_{t_{0}, t_{1}}\right)= & \left\{(y(t), \lambda, \mu(t), \xi(t)): t \in \Omega_{t_{0}, t_{1}}, y(t) \in C^{\infty}\left(\Omega_{t_{0}, t_{1}}, M\right), \lambda \in \mathbb{R}^{r},\right. \\
& \mu(t): \Omega_{t_{0}, t_{1}} \rightarrow \mathbb{R}^{\mathrm{msp}}, \xi(t): \Omega_{t_{0}, t_{1}} \rightarrow \mathbb{R}^{\mathrm{msp}} \\
& \text { verifying the constraints of (WDP) }\} .
\end{aligned}
$$

Consider $Y_{W}=\left\{y(t) \in C^{\infty}\left(\Omega_{t_{0}, t_{1}}, M\right):(y(t), \lambda, \mu(t), \xi(t)) \in \Delta_{W}\left(\Omega_{t_{0}, t_{1}}\right)\right\}$.

Theorem 5.1 (Weak duality) Let $x(\cdot)$ and $(y(\cdot), \lambda, \mu(\cdot), \xi(\cdot))$ be feasible solutions in problem (MVP) and its multitime multiobjective variational Wolfe dual problem (WDP), respectively. Suppose that the following hypotheses are satisfied: 
(a) $\int_{\gamma_{t_{0}, t_{1}}}\left\{\left\langle\lambda, f_{\alpha}\left(\pi_{y}(t)\right)\right\rangle+\left\langle\mu_{\alpha}(t), g\left(\pi_{y}(t)\right)\right\rangle+\left\langle\xi_{\alpha}(t), h\left(\pi_{y}(t)\right)\right\rangle\right\} d t^{\alpha}$ is strictly univex at point $y(\cdot)$ on $\Gamma\left(\Omega_{t_{0}, t_{1}}\right) \cup Y_{W}$ with respect to $\Phi, \eta$ and $b$,

(b) $a<0 \Longrightarrow \Phi(a)<0$ and $\Phi(0)=0$,

(c) $b(x(\cdot), y(\cdot))>0$.

Then the inequality $F(x(\cdot)) \leq \varphi(y(\cdot), \mu(\cdot), \xi(\cdot))$ is false.

Proof Let $x(\cdot)$ and $\left(y(\cdot), y_{\gamma}(\cdot), \lambda, \mu(\cdot), \xi(\cdot)\right)$ be feasible solutions in the considered multitime multiobjective variational problem (MVP) and the multitime variational Wolfe dual problem (WDP), respectively. Suppose, contrary to the result, that the inequality

$$
F(x(\cdot)) \leq \varphi(y(\cdot), \mu(\cdot), \xi(\cdot))
$$

holds. Thus, by the definition of $\varphi$, we have

$$
F^{i}(x(\cdot)) \leqq \int_{\gamma_{t_{0}, t_{1}}}\left\{f_{\alpha}^{i}\left(\pi_{y}(t)\right)+\left\langle\mu_{\alpha}(t), g\left(\pi_{y}(t)\right)\right\rangle+\left\langle\xi_{\alpha}(t), h\left(\pi_{y}(t)\right)\right\rangle\right\} d t^{\alpha}
$$

for $i=1, \ldots, r$ and

$$
F^{i^{*}}(x(\cdot))<\int_{\gamma_{t_{0}, t_{1}}}\left\{f_{\alpha}^{i^{*}}\left(\pi_{y}(t)\right)+\left\langle\mu_{\alpha}(t), g\left(\pi_{y}(t)\right)\right\rangle+\left\langle\xi_{\alpha}(t), h\left(\pi_{y}(t)\right)\right\rangle\right\} d t^{\alpha}
$$

for some $i^{*} \in\{1, \ldots, r\}$.

Multiplying (45) by $\lambda_{i}, i=1, \ldots, r$, and (46) by $\lambda_{i^{*}}$, we obtain, respectively,

$$
\lambda_{i} F^{i}(x(\cdot)) \leqq \int_{\gamma_{t_{0}, t_{1}}}\left\{\lambda_{i} f_{\alpha}^{i}\left(\pi_{y}(t)\right)+\lambda_{i}\left[\left\langle\mu_{\alpha}(t), g\left(\pi_{y}(t)\right)\right\rangle+\left\langle\xi_{\alpha}(t), h\left(\pi_{y}(t)\right)\right\rangle\right]\right\} d t^{\alpha}
$$

for $i=1, \ldots, r$ and

$$
\lambda_{i^{*}} F^{i^{*}}(x(\cdot))<\int_{\gamma_{t_{0}, t_{1}}}\left\{\lambda_{i^{*}} f_{\alpha}^{i^{*}}\left(\pi_{y}(t)\right)+\lambda_{i^{*}}\left[\left\langle\mu_{\alpha}(t), g\left(\pi_{y}(t)\right)\right\rangle+\left\langle\xi_{\alpha}(t), h\left(\pi_{y}(t)\right)\right\rangle\right]\right\} d t^{\alpha}
$$

for some $i^{*} \in\{1, \ldots, r\}$.

Using the feasibility of $x(\cdot)$ in problem (MVP) together with the constraint of (WDP) $\mu_{\alpha}(t) \geqq 0$, we get

$$
\left\langle\mu_{\alpha}(t), g\left(\pi_{x}(t)\right)\right\rangle+\left\langle\xi_{\alpha}(t), h\left(\pi_{x}(t)\right)\right\rangle \leqq 0 .
$$

By (47), (48) and (49), it follows that

$$
\begin{aligned}
& \int_{\gamma_{t_{0}, t_{1}}}\left\{\lambda_{i} f_{\alpha}^{i}\left(\pi_{x}(t)\right)+\lambda_{i}\left[\left\langle\mu_{\alpha}(t), g\left(\pi_{x}(t)\right)\right\rangle+\left\langle\xi_{\alpha}(t), h\left(\pi_{x}(t)\right)\right\rangle\right]\right\} d t^{\alpha} \\
& \leq \int_{\gamma_{t_{0}, t_{1}}}\left\{\lambda_{i} f_{\alpha}^{i}\left(\pi_{y}(t)\right)+\lambda_{i}\left[\left\langle\mu_{\alpha}(t), g\left(\pi_{y}(t)\right)\right\rangle+\left\langle\xi_{\alpha}(t), h\left(\pi_{y}(t)\right)\right\rangle\right]\right\} d t^{\alpha}
\end{aligned}
$$


for $i=1, \ldots, r$ and

$$
\begin{aligned}
& \int_{\gamma_{t_{0}, t_{1}}}\left\{\lambda_{i^{*}} f_{\alpha}^{i^{*}}\left(\pi_{x}(t)\right)+\lambda_{i^{*}}\left[\left\langle\mu_{\alpha}(t), g\left(\pi_{x}(t)\right)\right\rangle+\left\langle\xi_{\alpha}(t), h\left(\pi_{x}(t)\right)\right\rangle\right]\right\} d t^{\alpha} \\
& <\int_{\gamma_{t_{0}, t_{1}}}\left\{\lambda_{i^{*}} f_{\alpha}\left(\pi_{y}(t)\right)+\lambda_{i^{*}}\left[\left\langle\mu_{\alpha}(t), g\left(\pi_{y}(t)\right)\right\rangle+\left\langle\xi_{\alpha}(t), h\left(\pi_{y}(t)\right)\right\rangle\right]\right\} d t^{\alpha}
\end{aligned}
$$

for some $i^{*} \in\{1, \ldots, r\}$.

Adding both sides of (50) and (49) and taking into account the constraint of (WDP) $\langle\lambda, e\rangle=1$, we obtain

$$
\begin{gathered}
\int_{\gamma_{t_{0}, t_{1}}}\left\langle\lambda, f_{\alpha}\left(\pi_{x}(t)\right)\right\rangle+\left\langle\mu_{\alpha}(t), g\left(\pi_{x}(t)\right)\right\rangle+\left\langle\xi_{\alpha}(t), h\left(\pi_{x}(t)\right)\right\rangle d t^{\alpha} \\
\quad<\int_{\gamma_{t_{0}, t_{1}}}\left\{\left\langle\lambda, f_{\alpha}\left(\pi_{y}(t)\right)\right\rangle+\left\langle\mu_{\alpha}(t), g\left(\pi_{y}(t)\right)\right\rangle+\left\langle\xi_{\alpha}(t), h\left(\pi_{y}(t)\right)\right\rangle\right\} d t^{\alpha} .
\end{gathered}
$$

By hypotheses (b) and (c), (52) implies

$$
\begin{aligned}
& b(x(\cdot), y(\cdot)) \Phi\left(\int_{\gamma_{t_{0}, t_{1}}}\left\langle\lambda, f_{\alpha}\left(\pi_{x}(t)\right)\right\rangle+\left\langle\mu_{\alpha}(t), g\left(\pi_{x}(t)\right)\right\rangle+\left\langle\xi_{\alpha}(t), h\left(\pi_{x}(t)\right)\right\rangle d t^{\alpha}\right. \\
& \left.\quad-\int_{\gamma_{t_{0}, t_{1}}}\left\{\left\langle\lambda, f_{\alpha}\left(\pi_{y}(t)\right)\right\rangle+\left\langle\mu_{\alpha}(t), g\left(\pi_{y}(t)\right)\right\rangle+\left\langle\xi_{\alpha}(t), h\left(\pi_{y}(t)\right)\right\rangle\right\} d t^{\alpha}\right) \\
& \quad<0 .
\end{aligned}
$$

By Definition 3.1, it follows

$$
\begin{aligned}
\int_{\gamma_{t_{0}, t_{1}}} & \left\{\left\langle\eta\left(\pi_{x}(t), \pi_{y}(t)\right),\left\langle\lambda, \frac{\partial f_{\alpha}}{\partial y}\left(\pi_{y}(t)\right)\right\rangle+\left\langle\mu_{\alpha}(t), \frac{\partial g}{\partial y}\left(\pi_{y}(t)\right)\right\rangle\right.\right. \\
+ & \left.\left\langle\xi_{\alpha}(t), \frac{\partial h}{\partial y}\left(\pi_{y}(t)\right)\right\rangle\right\rangle+\left\langle D_{\gamma} \eta\left(\pi_{x}(t), \pi_{y}(t)\right),\left\langle\lambda, \frac{\partial f_{\alpha}}{\partial y_{\gamma}}\left(\pi_{y}(t)\right)\right\rangle\right. \\
+ & \left.\left.\left\langle\mu_{\alpha}(t), \frac{\partial g}{\partial y_{\gamma}}\left(\pi_{y}(t)\right)\right\rangle+\left\langle\xi_{\alpha}(t), \frac{\partial h_{\alpha}}{\partial y_{\gamma}}\left(\pi_{y}(t)\right)\right\rangle\right\rangle\right\} d t^{\alpha}
\end{aligned}
$$

$<0$.

For each $\alpha=1, \ldots, p$, we introduce

$$
V_{\alpha}\left(\pi_{y}(t), \lambda, \mu(t), \xi(t)\right)=\left\langle\lambda, f_{\alpha}\left(\pi_{y}(t)\right)\right\rangle+\left\langle\mu_{\alpha}(t), g\left(\pi_{y}(t)\right)\right\rangle+\left\langle\xi_{\alpha}(t), h\left(\pi_{y}(t)\right)\right\rangle
$$

Combining (54) and (55), we obtain

$$
\begin{aligned}
\int_{\gamma_{t_{0}, t_{1}}} & \left\{\left\langle\eta\left(\pi_{x}(t), \pi_{y}(t)\right), \frac{\partial V_{\alpha}}{\partial y}\left(\pi_{y}(t), \lambda, \mu(t), \xi(t)\right)\right\rangle\right. \\
+ & \left.\left\langle D_{\gamma} \eta\left(\pi_{x}(t), \pi_{y}(t)\right), \frac{\partial V_{\alpha}}{\partial y_{\gamma}}\left(\pi_{y}(t), \lambda, \mu(t), \xi(t)\right)\right\rangle\right\} d t^{\alpha}
\end{aligned}
$$

$<0$. 
The last part of the proof is similar to the proof of Theorem 4.1. Thus, in a similar manner as in the proof of Theorem 4.1, that is, by Lemma 2.1 we get a contradiction. Hence, the inequality $F(x(\cdot))<\varphi(y(\cdot), \lambda, \mu(\cdot), \xi(\cdot))$ is false.

Theorem 5.2 (Strong duality) Let $\bar{x}(\cdot)$ be a normal efficient solution of (MVP). Then there exist the vector $\bar{\lambda} \in \mathbb{R}^{r}$ and the smooth matrix functions $\bar{\mu}(t)=\left(\bar{\mu}_{\alpha}(t)\right): \Omega_{t_{0}, t_{1}} \rightarrow \mathbb{R}^{\mathrm{msp}}$ and $\bar{\xi}(t)=\left(\bar{\xi}_{\alpha}(t)\right): \Omega_{t_{0}, t_{1}} \rightarrow \mathbb{R}^{\mathrm{ksp}}$ such that $(\bar{x}(\cdot), \bar{\lambda}, \bar{\mu}(\cdot), \bar{\xi}(\cdot))$ is feasible in the Wolfe dual problem (WDP) and the objective functions of (MVP) and (WDP) are equal at these points. If also all the hypotheses of Theorem 5.1 are satisfied, then $(\bar{x}(\cdot), \bar{\lambda}, \bar{\mu}(\cdot), \bar{\xi}(\cdot))$ is a properly efficient solution in (WDP).

Proof Proof is similar to the proof of Theorem 4.2.

Proposition 5.1 Let $(\bar{y}(\cdot), \bar{\lambda}, \bar{\mu}(\cdot), \bar{\xi}(\cdot))$ be feasible in the Wolfe multitime multiobjective variational problem (MWDP) and $\bar{y}(\cdot) \in \Gamma\left(\Omega_{t_{0}, t_{1}}\right)$. Further, assume that the following hypotheses are satisfied:

(a) $\int_{\gamma_{t_{0}, t_{1}}}\left\{\left\langle\lambda, f_{\alpha}\left(\pi_{y}(t)\right)\right\rangle+\left\langle\mu_{\alpha}(t), g\left(\pi_{y}(t)\right)\right\rangle+\left\langle\xi_{\alpha}(t), h\left(\pi_{y}(t)\right)\right\rangle\right\} d t^{\alpha}$ is strictly univex at the point $\bar{y}(\cdot)$ on $\Gamma\left(\Omega_{t_{0}, t_{1}}\right) \cup Y_{W}$ with respect to $\Phi, \eta$ and $b$,

(b) $a<0 \Longrightarrow \Phi(a)<0$ and $\Phi(0)=0$,

(c) $b(x(\cdot), y(\cdot))>0$.

Then $\bar{y}(\cdot)$ is a properly efficient solution in problem (MVP).

Theorem 5.3 (Converse duality) Let $(\bar{y}(\cdot), \bar{\lambda}, \bar{\mu}(\cdot), \bar{\xi}(\cdot))$ be a properly efficient solution in the Wolfe dual problem (WDP) and $\bar{y}(\cdot) \in \Gamma\left(\Omega_{t_{0}, t_{1}}\right)$. Further, assume that the following hypotheses are satisfied:

(a) $\int_{\gamma_{t_{0}, t_{1}}}\left\{\left\langle\lambda, f_{\alpha}\left(\pi_{y}(t)\right)\right\rangle+\left\langle\mu_{\alpha}(t), g\left(\pi_{y}(t)\right)\right\rangle+\left\langle\xi_{\alpha}(t), h\left(\pi_{y}(t)\right)\right\rangle\right\} d t^{\alpha}$ is univex at the point $\bar{y}(\cdot)$ on $\Gamma\left(\Omega_{t_{0}, t_{1}}\right) \cup Y_{W}$ with respect to $\Phi, \eta$ and $b$,

(b) $a<0 \Longrightarrow \Phi(a)<0$ and $\Phi(0)=0$,

(c) $b(x(\cdot), y(\cdot))>0$.

Then $\bar{y}(\cdot)$ is a properly efficient solution in the considered multitime multiobjective variational problem (MVP).

\section{Concluding remarks}

In this research paper, a new class of nonconvex multitime variational problems has been considered. We have defined the concept of univexity for a path-independent curvilinear vector functional as a generalization of a vector-valued univex function. The so-called univex functions unify many various classes of generalized convex concepts in optimization theory. Therefore, the sufficient optimality conditions for proper efficiency and several duality theorems in the sense of Mond-Weir and in the sense of Wolfe, which have been established in the paper, for a new class of nonconvex multitime multiobjective variational problems extend adequate results already existing in optimization theory. 


\section{Author details}

${ }^{1}$ Faculty of Applied Sciences, University 'Politehnica' of Bucharest, Splaiul Independenţei, No. 313, Bucharest, 060042, Romania. ${ }^{2}$ Faculty of Mathematics and Computer Science, University of Łódź, Banacha 22, Łódź, 90-238, Poland.

Received: 20 March 2014 Accepted: 31 July 2014 Published: 2 September 2014

\section{References}

1. Chinchuluun, A, Pardalos, PM: A survey of recent developments in multiobjective optimization. Ann. Oper. Res. 154, 29-50 (2007)

2. Aghezzaf, B, Khazafi, K: Sufficient conditions and duality for multiobjective variational problems with generalized B-invexity. Control Cybern. 33, 113-126 (2004)

3. Ahmad, I, Sharma, S: Sufficiency and duality for multiobjective variational control problems with generalized $(F, \alpha, \rho, \theta)-V$-convexity. Nonlinear Anal. 72, 2564-2579 (2010)

4. Arana-Jiménez, M, Rufián-Lizana, A, Ruiz-Garzón, G, Osuna-Gómez, R: Efficient solutions in V-KT-pseudoinvex multiobjective control problems: a characterization. Appl. Math. Comput. 215, 441-448 (2009)

5. Bector, CR, Husain, I: Duality for multiobjective variational problems. J. Math. Anal. Appl. 166, 214-229 (1992)

6. Bhatia, D, Mehra, A: Optimality conditions and duality for multiobjective variational problems with generalized B-invexity. J. Math. Anal. Appl. 234, 314-360 (1999)

7. Hachimi, M, Aghezzaf, B: Sufficiency and duality in multiobjective variational problems with generalized type I functions. J. Glob. Optim. 34, 191-218 (2006)

8. Mishra, SK, Mukherjee, RN: On efficiency and duality for multiobjective variational problems. J. Math. Anal. Appl. 187, 40-54 (1994)

9. Nahak, C, Nanda, S: Duality for multiobjective variational problems with invexity. Optimization 36, $235-248$ (1996)

10. Nahak, C, Nanda, S: On efficiency and duality for multiobjective variational control problems with $(F, \rho)$-convexity. J. Math. Anal. Appl. 209, 415-434 (1997)

11. Nahak, C, Nanda, S: Sufficient optimality criteria and duality for multiobjective variational control problems with V-invexity. Nonlinear Anal. 66, 1513-1525 (2007)

12. Chinchuluun, A, Pardalos, PM, Migdalas, A, Pitsoulis, L (eds.): Pareto Optimality, Game Theory and Equilibria. Springer, New York (2008)

13. Udrişte, C, Tevy, I: Multitime Euler-Lagrange-Hamilton theory. WSEAS Trans. Math. 6, 701-709 (2007)

14. Pitea, A, Udrişte, C, Mititelu, Ş: PDI\&PDE-constrained optimization problems with curvilinear functional quotients as objective vectors. Balk. J. Geom. Appl. 14(2), 75-88 (2009)

15. Pitea, A, Udrişte, C, Mititelu, Ş: New type dualities in PDI and PDE constrained optimization problems. J. Adv. Math. Stud. 2(2), 81-90 (2009)

16. Bector, CR, Suneja, SK, Gupta, S: Univex functions and univex nonlinear programming. In: Proceedings of the Administrative Sciences Association of Canada, pp. 115-124 (1992)

17. Hanson, MA: On sufficiency of the Kuhn-Tucker conditions. J. Math. Anal. Appl. 80, 545-550 (1981)

18. Antczak, T: An $\eta$-approximation approach in nonlinear vector optimization with univex functions. Asia-Pac. J. Oper. Res. 23, 525-542 (2006)

19. Popa, M, Popa, M: Mangasarian type higher-order duality for a class of nondifferentiable mathematical programming Rev. Roum. Math. Pures Appl. 49(2), 163-172 (2004)

20. Mishra, SK, Rueda, N, Giorgi, NG: Multiobjective programming under generalized type I univexity. An. Univ. Bucur., Mat. LII(2), 207-224 (2003)

21. Khazafi, K, Rueda, N: Multiobjective variational programming under generalized type I univexity. J. Optim. Theory Appl. 142, 363-376 (2009)

22. Udrişte, C, Dogaru, O, Ţevy, I: Null Lagrangian forms and Euler-Lagrange PDEs. J. Adv. Math. Stud. 1(1-2), 143-156 (2008)

23. Geoffrion, AM: Proper efficiency and the theory of vector maximization. J. Math. Anal. Appl. 22, 618-630 (1968)

doi:10.1186/1029-242X-2014-333

Cite this article as: Pitea and Antczak: Proper efficiency and duality for a new class of nonconvex multitime multiobjective variational problems. Journal of Inequalities and Applications 2014 2014:333.

\section{Submit your manuscript to a SpringerOpen ${ }^{\ominus}$ journal and benefit from:}

- Convenient online submission

Rigorous peer review

- Immediate publication on acceptance

Open access: articles freely available online

- High visibility within the field

- Retaining the copyright to your article 